\title{
ANÁLISE QUALITATIVA DAS PERCEPÇÕES DE CIRURGIÕES- DENTISTAS ENVOLVIDOS NOS ATENDIMENTOS DE PACIENTES COM NECESSIDADES ESPECIAIS DE SERVIÇOS PÚBLICOS MUNICIPAIS
}

\author{
QUALITATIVE ANALYSIS OF DENTISTS' PERCEPTIONS \\ INVOLVED IN PATIENT CARE WITH SPECIAL NEEDS FROM \\ MUNICIPAL SERVICES
}

\author{
Alexandre Luiz Affonso Fonseca ${ }^{1}$ \\ Ligia Ajaime Azzalis ${ }^{2}$ \\ Fernando Luiz Affonso Fonseca ${ }^{3}$ \\ Carlos Botazzo ${ }^{4}$
}

Fonseca, ALA et al. Análise qualitativa das percepções de cirurgiões-dentistas envolvidos nos atendimentos de pacientes com necessidades especiais de serviços públicos municipais. Rev Bras Crescimento Desenvolvimento Hum. 2010; 20(2): 208-216

\section{Resumo}

O número de pessoas com necessidades especiais bem como as questões referentes ao atendimento desses pacientes e a partir da resolução 25/2002, publicada no Diário Oficial da União - D.O.U. - em 28/05/2002 pelo Conselho Federal de Odontologia, regulamenta a especialidade, com intenção de capacitar os cirurgiões-dentistas ao atendimento de pessoas que necessitam de cuidados odontológicos especiais durante toda a sua vida ou por um período. Um dos pontos levantados no atendimento a esses pacientes está a questão da integralidade de modo que essa seria a de compreender o paciente e propor atendê-lo por inteiro em suas necessidades de saúde. Assim, o objetivo é verificar as percepções dos cirurgiões-dentistas sobre o atendimento de crianças com necessidades especiais. Desta maneira, evidenciaram-se dificuldades desses profissionais em descrever as reais necessidades das crianças com necessidades especiais no tocante ao atendimento odontológico, tanto em nível do serviço de saúde público municipal, como nos quesitos de formação específica para o exercício profissional com esta população.

Palavras-chave: Pessoas portadoras de necessidades especiais; serviços de saúde bucal; assistência odontológica para pessoas portadoras de necessidades especiais; avaliação em saúde/ classificação; promoção da saúde.

\footnotetext{
Programa de Pós - Graduação em Ciências da Coordenadoria de Controle de Doenças da Secretaria de Estado da Saúde de São Paulo

2 Professor Adjunto da Universidade Federal de São Paulo (UNIFESP-Diadema). Professor Adjunto da Universidade Federal de São Paulo (UNIFESP-Diadema).

4 Professor da Faculdade de Odontologia da Universidade de São Paulo (FOUSP).

Autor: Alexandre Luiz Affonso Fonseca. - Endereço para correspondência: Alameda dos Anapurus, no. 746, apto. 14. - Moema. São Paulo - SP - CEP: 04087-002 - E-mail: aleluiz2004@hotmail.com

Baseado na Dissertação de Mestrado de Alexandre Luiz Affonso Fonseca com o título: "Relação entre o perfil do paciente com necessidade especial assistido em serviços públicos de saúde e os limites de atuação do cirurgião-dentista”. Dissertação apresentada ao Programa de Pós - Graduação em Ciências da Coordenadoria de Controle de Doenças da Secretaria de Estado da Saúde de São Paulo, para obtenção do título de Mestre em Ciências, Área de Concentração: Infectologia em Saúde Pública, São Paulo 2008.
} 


\begin{abstract}
The large number of people with special needs as well as issues concerning the care of these patientsresulted in the Resolution 25/2002, published in the Official Gazette of the Union - On 28/05/2002 by the Federal Council of Dentistry; it regulates the specialty, with the intent to enable dentists to take care for people in need of special dental care throughout their life or a period. One of the points raised in response to these patients is the issue of totality to understand the patient and propose to serv hin or her in all their health's needs. This paper aims to draw from a qualitative analysis, the perceptions of dentists responsible for these consultations in five municipalities of greater Sao Paulo. It is evident that the professionals' difficulties in describing what really is inherent with the patient in special need of dental care which is itself an health municipal public service and also what are their own limits facing the attendants described. It was concluded that recognition of these limits is a key factor that they can be overcome in order to glimpse the care.
\end{abstract}

Key words: disabled persons; dental health services; dental care for disabled; health evaluation/classification; health promotion.

\section{INTRODUÇÃO}

Uma pessoa com necessidades especiais, de forma geral, sofre com o descaso da sociedade, que se mostra, muitas vezes, despreparada, preconceituosa e inapta para atender às necessidades deste indivíduo ${ }^{1}$. Entretanto, por vezes é nesta mesma sociedade que surgem idéias transformadoras visando à aceitação destes indivíduos no grupo a que pertencem, com o conseqüente resgate da dignidade e, dentro do possível, da autonomia ${ }^{2}$.

É reconhecida a importância da inclusão social destas pessoas, que precisam de cuidados especiais na educação e na saúde. Assim, torna-se necessária uma integralização das ações, caracterizada pelo conjunto de trabalhos de uma equipe multidisciplinar, objetivando a reabilitação e a inclusão da pessoa com necessidades especiais ${ }^{3}$.

Apesar de estar claro na Constituição do Brasil de 1988 que a saúde é um direito de todos e um dever do Estado ${ }^{4}$, observa-se que a atenção à pessoa com necessidades especiais vem sendo prestada de forma predominantemente irregular e antidemocrática, contando muito com a ajuda de trabalhos voluntários². Assim, a implantação do Sistema Único de Saú- de (SUS), garante a assistência integral em ações preventivas, curativas e reabilitadoras independente da condição especial do cidadão ${ }^{5}$.

A questão da integralidade, que seria a de compreender o paciente e propor atendê-lo por inteiro em suas necessidades de saúde, é evidente e de suma importância no atendimento às pessoas com necessidades especiais. Portanto, a prática clínica integral é desejável como ideal para a equipe de saúde bucal porque o trabalho do dentista é naturalmente fragmentado. No entanto, a necessidade do especialista e do cuidado integral teria que enfrentar a ressignificação do ato clínico e depois a ressignificação da relação profissional-paciente ${ }^{6}$.

Segundo Figueiredo (2002), na Odontologia utiliza-se agora o conceito "pacientes com necessidades especiais”. Em nível internacional, a International Association for Disability and Oral Health (IADH) inclui, além dos portadores de deficiência, pessoas com restrições de capacidades advindas da idade ou de agravos adquiridos, os quais limitam a função intelectual, física ou social - essa associação atua no incenti- 
vo ao desenvolvimento da pesquisa, educação e tratamento da saúde bucal de pessoas com condições incapacitantes ${ }^{7}$.

A partir da resolução 25/2002, publicada no Diário Oficial da União em 28/05/2002 pelo Conselho Federal de Odontologia, regulamentou-se a especialidade, com intenção de capacitar os cirurgiões-dentistas ao atendimento de pessoas que necessitam de cuidados odontológicos especiais durante toda a vida ou por um período ${ }^{8}$.

A partir de então, o cirurgião-dentista especialista passa a ser também um reabilitador deste paciente com necessidade especial e deverá mudar paradigmas para melhorar a qualidade de vida destes, por meio de tratamentos preconizados pela odontologia convencional e de estudos multidisciplinares desenvolvidos com outras especialidades. É uma especialidade, portanto, que permite ao cirurgião-dentista especialista adquirir "sensibilidade social". Tal procedimento retira-o da frieza dos métodos em que o tecnicismo é produzido e reproduzido, permitindo que este profissional seja habilitado a atender pessoas, respeitando limites, condições especiais e visando ao atendimento integral ${ }^{9}$.

Assim, o objetivo é verificar as percepções dos cirurgiões-dentistas sobre o atendimento de crianças com necessidades especiais.

\section{MÉTODO}

A presente pesquisa é qualitativa e foi realizada em duas etapas. $\mathrm{Na}$ primeira etapa foram feitos estágios observacionais em cinco municípios, que pertenciam à extinta DIRV da Secretaria da Saúde do Estado de São Paulo, e apresentam atendimento odontológico para pacientes com necessidades especiais. Foram incluídos na pesquisa os municípios de Barueri, Carapicuíba, Embu das Artes, Itapecerica da Serra e Santana do Parnaíba. No processo de investigação, o pesquisador entrava em contato com os coordenadores de saúde bucal de cada município incluído na pesquisa, buscava o contato com o cirurgião-dentista responsável pelo atendimento e marcava a visita para a realização do estágio observacional.

Os estágios observacionais tinham como finalidade conhecer os serviços de odontologia que atendiam os pacientes com necessidades especiais seguindo um roteiro de observação. Nesse roteiro, o pesquisador preocupava-se com fatores que caracterizam um serviço público de saúde como: estruturação, fluxo, sistema de referência e contra-referência, a existência de um protocolo de atendimento e /ou referenciamento, o tipo de serviço ofertado, a facilidade de acesso ao serviço, existência de demanda reprimida além da disponibilidade de material de consumo.

Na segunda etapa, foram realizadas cinco entrevistas orientadas por meio de questionários aos cirurgiões-dentistas responsáveis por estes atendimentos. Os profissionais eram entrevistados sobre a capacidade de conseguir suprir as necessidades odontológicas do paciente atendido, respaldo por parte do serviço, pacientes com facilidade ou não de atendimento, demais dificuldades encontradas no atendimento e determinação de limites pessoais e do serviço. Tais perguntas eram determinantes para que se pudesse traçar o perfil desses dentistas envolvidos nos atendimentos, bem como a capacidade de oferecer subsídios para determinar fatores que pudessem colaborar para determinação dos limites.

Para garantir o sigilo dos profissionais entrevistados bem como preservar a descrição e a identidade dos serviços mediante a observação realizada, os resultados das entrevistas foram tratados segundo análise qualitativa, sem identificação dos entrevistados, conforme exposto no Termo de Consentimento Livre Esclarecido.

Após a realização do estágio, que durou de duas a três visitas, dependendo da disponibilidade do serviço a ser avaliado, foi feita a entrevista com o cirurgião-dentista. É válido 
esclarecer que não se tratava, verdadeiramente, de estágio, e sim de observação total, em que o pesquisador observava os eventos que se desenrolaram na situação em que se encontrava, sem, no entanto, propor ou desenvolver intervenções de quaisquer naturezas.

Após esta etapa, era feita a entrevista pelo próprio pesquisador, que transcrevia os resultados em um rascunho, para depois reescrevê-los de forma definitiva e enviá-los para o entrevistado para possíveis alterações, modificações ou até correções.

Isso foi proposto pelo pesquisador aos sujeitos da pesquisa para evitar que se sentissem avaliados durante a realização do estágio observacional e entrevista. Os entrevistados puderam ter, então, participação efetiva e prestimosa, podendo agir e falar à vontade durante os contatos com o pesquisador. Dos cinco serviços descritos, em dois deles, os sujeitos da pesquisa acabaram modificando as transcrições feitas pelo pesquisador.

A expressão mais comumente usada para representar o tratamento dos dados de uma pesquisa qualitativa é a Análise de Conteúdo ${ }^{10}$. Este termo não significa só um procedimento técnico, mas também faz parte de uma história que busca teoria e prática no campo das investigações sociais, sendo definida segundo Bardin (1979) como: "Um conjunto de técnicas de análise de comunicação visando obter, por procedimentos sistemáticos e objetivos de descrição do conteúdo das mensagens, indicadores (quantitativos ou não) que permitam a inferência de conhecimentos relativos às condições de produção/recepção destas mensagens ${ }^{11}$.”

Como um dos objetivos deste trabalho foi relatar os limites de atuação dos cirurgiões-dentistas envolvidos em serviços públicos de odontologia que atendem pacientes com necessidades especiais, a presença do pesquisador in loco era fundamental para avaliar o ambiente e as circunstâncias em que estes profissionais atuam, e como sugere Alves ${ }^{12}$, sem deixar-se determinar pelas imagens ou idéias preconcebidas, pois descobertas podem surpreender ${ }^{12}$.

Em se tratando da avaliação ética da pesquisa, o protocolo de pesquisa foi encaminhado ao Comitê de Ética em Pesquisa do Instituto de Saúde da Secretaria da Saúde do Estado de São Paulo (CEPIS) e este, por meio dos seus relatores, sugeriu melhorias no Termo de Consentimento Livre Esclarecido (TCLE) originalmente apresentado, principalmente na questão do sigilo dos participantes da pesquisa bem como dos serviços descritos, sendo aprovado pelo Comitê em 29/06/2006 (Protocolo $n^{0}$ 005/06)

\section{RESULTADOS}

Os resultados apresentados correspondem a recortes das falas transcritas pelo pesquisador e obtidas por análise de conteúdo com correção prévia dos profissionais entrevistados.

Sobre o cirurgião-dentista conseguir suprir as necessidades dos pacientes com necessidades especiais.

Seguem as falas dos profissionais:

"Não na sua totalidade, quando há necessidade de sedação endovenosa ou tratamento sob anestesia geral, como não há no município este atendimento, acabo encaminhando para hospitais de São Paulo como o Hospital das Clínicas e Hospital do Servidor Público Estadual. Essas referências são informais e feitas pelo meu próprio conhecimento". (grifos do pesquisador)

"Sim, embora neste momento não esteja ocorrendo atendimento odontológico para pacientes com doenças neuromotora, necessidades especiais mentais, visuais, auditivas e para os sindrômicos”. (grifos do pesquisador)

"De jeito nenhum. Existe uma dificuldade muito grande na obtenção dos resultados positivos principalmente em relação à prevenção". (grifos do pesquisador) 
“Não, faço tudo o que está no meu alcance. Às vezes existem pacientes que precisam de ortodontia e acabam sendo encaminhados". (grifos do pesquisador)

"Sim, em se tratando de atendimento para pacientes com necessidades especiais é possível à realização de todos os procedimentos que o paciente permitir receber. Quando houver necessidade e oportunidade de encaminhamento este é feito para as outras especialidades do CEO”. (grifos do pesquisador)

Em se tratando de maiores dificuldades em relação ao atendimento $x$ pacientes que acham mais fáceis e mais difíceis de serem atendidos.

Seguem as explanações:

"Qualquer paciente que colabore com o tratamento, é fácil de atender. O que tenho mais dificuldades são os autistas, principalmente os de idade avançada”.

"Os pacientes com doenças infectocontagiosas acabam sendo os mais fáceis para atender, isso porque possuo um bom contato com médicos que os encaminham. Os mais difíceis acabam sendo os pacientes com doenças sistêmicas crônicas, porque sempre chegam descompensados e o contato com o médico endocrinologista é muito difícil, justamente pelo fato de não estarem disponibilizados no mesmo local onde é realizado o atendimento, como ocorre com os pacientes que vêem encaminhados do serviço DST/AIDS”. (grifos do pesquisador)

"Os mais difíceis são os pacientes portadores de autismo. Eles são a pedra do meu sapato (rs).......para um pouco e diz: é um mistério atendê-los, não é? Acho que os pacientes com problemas sistêmicos, devido não terem comprometimento intelectual, são os mais fáceis de atender. Eles deitam, se posicionam corretamente na cadeira (odontológica), têm compreensão, têm autonomia e têm resposta positiva ao tratamento". (grifos do pesquisador)

"Talvez os deficientes mentais leves ou moderados, não sei, talvez pelo fato de que qualquer dentista poderia atendê-los, ele apresentam uma certa compreensão, ficam mais dinâmicos os atendimentos". (grifos do pesquisador)

"Os mais difíceis são alguns autistas. O trabalho tem que ser mais rápido, o tempo na cadeira deles é menor. Eu saio mais estressada. Houve um maior desgaste". (grifos do pesquisador)

"O paciente mais fácil é o com síndrome de down e o mais difícil é o autista. Fico super estimulada quando vejo que o paciente é síndrome de down, mas com autista, ai é fogo, sabia? Eu, na real, nunca consegui atender nenhum! É super frustrante........". (grifos do pesquisador)

Sobre os limites do profissional $x$ limites do serviço

Observem as declarações dadas sobre os limites dos serviços pesquisados e os limites individuais dos profissionais envolvidos nos atendimentos dos pacientes com necessidades especiais:

"Para mim não há limites, sempre há tentativas mesmo que eles (pacientes) resistam, eu tento sanar as necessidades que eles apresentam. Quanto ao serviço também, mesmo que eu não consiga encaminhar alguns deles (pacientes) para o CEO ou que o especialista não consiga fazer o que eu pedi, tento junto com a coordenação solucionar o problema...pausa e depois continua: Acho sempre que é minha responsabilidade!” (grifos do pesquisador)

"Sempre há tentativas, não existem limites, sempre experimento. São diversas tentativas até saber se existem condições, se não houver acabo encaminhado... pára um pouco e, suspirando, completa: acredito que cheguei nesta situação porque houve acomodação da minha parte, nem peço mais nada e sempre acabo fazendo. Já atendi sem foco de luz. Sabe tenho dó de dispensar o paciente e acabo atendendo". (grifos do pesquisador)

"Quando preciso de muita gente para conter/segurar o paciente, que mesmo depois 
de várias sessões de dessensibilização ainda tenho que conter, até porque isso compromete o resultado técnico do procedimento odontológico, para mim isso é um fator limitante. Eu fico com dor, dor física mesmo, sabe às vezes tenho que adiar algumas coisas que tenho que fazer neles por não ter condições físicas de atender sabia? Sobre o serviço, além de tudo que já conversamos, acho que a limitação fica por conta dos pacientes de alto risco, de complicações que podem acontecer, porque não há suporte médico para situações emergenciais". (grifos do pesquisador)

"O limite é estabelecido por cada paciente. Por exemplo: um paciente que necessita de uma cirurgia Le Fort I é lógico que não consigo fazer este tipo de atendimento, mas dentro da rotina do meu atendimento ambulatorial eu não tenho limites, sobre este serviço município não é limitado. Atualmente o que não atendemos são os pacientes com doenças neuromotoras, necessidades especiais e síndromes. Só atendo pacientes nestas condições quando estiverem em quadro de urgência”. (grifos do pesquisador)

"Os limites de atendimento para mim são para casos extremos onde não é possível o atendimento ambulatorial de patologias específicas em que é necessário o encaminhamento para atendimento em nível hospitalar, seja sob sedação ou anestesia geral. A dificuldade de encaminhamento para serviços específicos acaba sendo uma grande limitação do serviço prestado, mesmo que eu tenha uma liberdade grande de trabalho e não haja problemas quanto aos materiais de consumo". (grifos do pesquisador)

\section{DISCUSSÃO}

Verificou-se que quando os profissionais eram entrevistados sobre conseguir suprir todas as necessidades dos pacientes com neces- sidades especiais, esperava-se, que esses pudessem reconhecer que além de dentistas, também são seres humanos, e que como seres humanos também apresentam dificuldades particulares, medos, ansiedades e expectativas sobre os atendimentos que realizam.

Muitas vezes, o fato de não suprir todas as necessidades do paciente em questão pode não estar diretamente relacionado com as dificuldades inerentes aos serviços ou até mesmo com as necessidades especiais dos pacientes. Essas dificuldades podem estar relacionadas com os próprios profissionais, e não dificuldades na técnica odontológica, mais sim em toda a complexidade que exige um atendimento para pessoas com necessidades especiais.

Essas dificuldades podem estar relacionadas às questões humanas, questões de ordem moral, filosófica e psicológica, e também, em não conseguir suprir as necessidades dos pacientes. Isso pôde ser visto durante os estágios observacionais, na realização dos atendimentos, porém representadas de outra forma, e não foi evidenciada, nem reconhecida por nenhum dos sujeitos da pesquisa.

Somente na terceira declaração, o profissional levou em consideração o fato de que o atendimento para pacientes com necessidades especiais é extremamente complexo. Na explanação: "na dificuldade de obtenção de resultados positivos", no fato também de que, muitas vezes, como profissionais, talvez não seja possível a realização do tratamento ideal, mas sim daquele que foi possível ser realizado, ou seja, o tratamento real.

Este ponto deverá ser trabalhado pelo profissional, porque da mesma forma que o paciente possui expectativas sobre o tratamento proposto, o profissional, como proponente deste tratamento, também tem essas expectativas.

Portanto, o fato de não conseguir suprir as necessidades dos pacientes não poderá estar vinculado somente à questão da prática e técnica odontológica bem como com uma estruturação perfeita de serviços, mas também 
deverá estar relacionada a questões e dificuldades de ordem moral, filosófica e psicológica dos profissionais.

Também fica clara a necessidade do envolvimento dos familiares e/ou cuidadores e profissionais para o desenvolvimento de medidas preventivas e educativas específicas para os pacientes com necessidades especiais, mesmo que isso seja caracterizado como muito difícil e ainda, evidenciado e confirmado na declaração do profissional ${ }^{13}$.

Os demais profissionais entrevistados somente levaram em consideração a questão dos encaminhamentos, como se todas as dificuldades em atender pacientes especiais dependessem somente de um sistema de referência e contra-referência que fosse efetivo.

Portanto, desconsideram a deficiência do paciente envolvido, as dificuldades impostas pelas necessidades especiais e até mesmo as dificuldades inerentes ao profissional que trabalha num serviço público municipal, com alta demanda de pacientes e baixo controle na regulação destes serviços.

É necessário que estes profissionais sejam também preparados para lidar com as suas próprias dificuldades, porque se conseguirmos, no futuro, uma estruturação de serviços próxima do ideal, ainda existirão dificuldades e não será possível suprir todas as necessidades dos pacientes e a causa disso pode estar relacionada com as dificuldades individuais dos profissionais.

Sobre essa questão, ainda evidencia-se a dificuldade em reconhecer a figura humana em detrimento da técnica odontológica, ou seja, o tecnicismo odontológico representado e exigido no campo clínico como sendo a "chave" do sucesso no tratamento.

Isso também foi visto na forma como classificaram os pacientes que são mais fáceis e aqueles que são mais difíceis, ficando implícita uma predileção em atender pacientes que possam se comportar como os nossos manequins odontológicos da época da graduação, ou seja, que seja possível a reprodução da técnica odontológica sem a interferência do paciente.

Mesmo alguns deles sendo especialistas no atendimento de pacientes especiais, ainda mostram-se mais à vontade ao atender pacientes que se comportam de modo mais calmo, e são mais dóceis no consultório odontológico, como os pacientes com Síndrome de Down ${ }^{14}$.

Mais uma vez, em todas as citações, fica expressa a facilidade de comunicação de determinados pacientes para com os profissionais, sendo caracterizado como os de mais fácil atendimento os que possuem maior facilidade de compreensão e que possam responder melhor aos atendimentos.

Todos os profissionais foram unânimes em dizer que os autistas apresentam maiores dificuldades nos atendimentos, talvez pelo fato de o autismo ser uma síndrome definida por alterações presentes desde idades muito precoces, tipicamente antes dos três anos de idade, e que se caracteriza sempre por desvios qualitativos na comunicação, na interação social e no uso da imaginação ${ }^{15}$.

Essa tríade presente no paciente autista ainda é responsável por um padrão de comportamento restrito e repetitivo, mas com condições de inteligência que podem variar do retardo mental a níveis acima da média ${ }^{15}$.

Portanto, para uma profissão que ainda é muito baseada no tecnicismo dos procedimentos, despersonificando o paciente, torna-se extremamente difícil para os profissionais a realização do atendimento, principalmente para este público em questão.

Alguns profissionais citam o fato do bom contato com as outras áreas, também responsáveis pelos cuidados em saúde desses pacientes, ser um facilitador do atendimento, caracterizando a multidisciplinaridade recomendada por Seger et al ${ }^{16}$, que ressaltam a importância da atuação da equipe multidisciplinar, fazendo com que a especificidade do trabalho seja realizada em conjunto com diferentes profissio- 
nais nas diversas áreas afins visando à reabilitação do paciente ${ }^{16}$.

Isso é evidenciado nas falas dos sujeitos quando indagados sobre os limites de atuação desses profissionais, que aparecem muitas vezes sendo confundidos e mal-interpretados por parte deles, ou seja, o que poderia ser um limite individual do profissional muitas vezes é atribuído ao serviço e o contrário também foi observado.

Nas duas últimas declarações, o profissional limita seu atendimento à custa de um sistema de referência e contra-referência que não é efetivo, ressaltando a dificuldade dos encaminhamentos para atendimento sob anestesia geral e chega a uma situação de extrema especificidade ao citar: "um paciente que necessita de uma cirurgia Le Fort I é lógico que não consigo fazer este tipo de atendimento”.

$\mathrm{Na}$ terceira declaração, o profissional demonstra certo grau de estresse em relação aos seus atendimentos e observa-se ainda preocupação com a técnica odontológica.

Isso fica evidente quando refere dor física e adiamento na realização de certos procedimentos em determinados pacientes que possam desgastá-lo fisicamente. Ainda refere, como limite do serviço, a falta de suporte médico em situações de emergências que possam ocorrer no atendimento de pacientes de alto risco, demonstrando até certa insegurança. Fica implícito um sofrimento psíquico por parte do profissional.

Na segunda declaração, o profissional embora demonstre ser persistente quanto às suas tentativas, revela certo grau de acomodação e insatisfação. De modo que a declaração nos remete a um desabafo por parte do profissional em relação ao seu descontentamento.

Na primeira declaração fica evidente que o profissional é um entusiasta e muito persistente nos seus atendimentos, mostrando-se preocupado em resolver os problemas que lhe são apresentados e ainda deixa claro: "Acho sempre que é minha responsabilidade!”, ou seja, não medindo esforços para sanar as questões, demonstrando envolvimento nos atendimentos.

Embora sejam evidenciadas situações de extrema especificidade pelo profissional que acabam por determinar o seu limite em relação ao atendimento, ou casos em que o profissional afirma não possuir limites em relação ao mesmo, há uma grande lacuna entre o que é limite dos serviços e quais são os limites individuais dos profissionais.

Neste caso, valem outras considerações e caberiam outras perguntas para que estes profissionais pudessem estabelecer esses limites. Será que é possível a realização de uma restauração de classe I de amálgama em um paciente com autismo? Se estes profissionais, porventura, não conseguirem realizar tal restauração, isso poderá ser interpretado por eles como um limite profissional ou um limite do serviço?

Observem como foram confundidos o estabelecimento e atribuições de limites entre os serviços em questão e os limites dos profissionais; ora, se não existem limites para muitos deles, ou se o serviço não é limitado de alguma forma, então por que há dificuldade em atender pacientes com desvios de comportamento como os autistas? Essa dificuldade não é imposta pelo serviço e muito menos pela pessoa que é autista e sim pelo profissional que deveria reconhecer seus limites nas questões referentes ao atendimento de pessoas com necessidades especiais.

O reconhecimento de limites é um fator fundamental para que estes possam ser superados e para que se vislumbre o atendimento integral. Integralidade esta não somente dentro do campo odontológico, mas aquela que atinja as necessidades e expectativas do ser humano especial na sua totalidade.

Portanto, é necessário realmente mudar o "olhar" em todos os sentidos e reconhecer a necessidade dos serviços públicos de saúde disponíveis serem efetivos, permitindo que as ações de saúde alcancem o processo inclusivo dessas pessoas com deficiência. 


\section{REFERÊNCIAS}

1. O'Donnell D. The special needs patient treatment in general practice: is it feasible? International Dental Journal 1996; 24(04): 315-319.

2. Castilho LS, Carvalho CF, Toso FP, Jacob MF, Abreu MHNG, Resende VLS. Utilização do INTO para triagem de grandes grupos populacionais Experiência com pacientes especiais. Revista do CROMG 2000; 6(3): 195-199.

3. BRASIL. Ministério da Saúde. Atenção à pessoa portadora de deficiência no sistema único de saúde. Brasília: 1993. 48p.

4. BRASIL. Constituição da República Federativa do Brasil, promulgada em 5 de outubro de 1988. Rio de Janeiro: Edições Trabalhistas, 1988. p 149.

5. BRASIL. Lei Número 8080 - 19 de set. 1990. Dispõe sobre as condições para a promoção, proteção e recuperação da saúde, a organização e o funcionamento aos serviços correspondentes e dá outras providências. Diário Oficial da União, Brasília, 20 de set. 1990.

6. Botazzo C. Novas Abordagens em saúde bucal. A questão da integralidade. In: Garcia DM (Org.). Novos rumos da saúde bucal: os caminhos da integralidade. Rio de Janeiro; ABO/ANS/UNESCO, 2005, p. 43-7.

7. Figueiredo JR. Estratégias para a provisão de cuidados no atendimento odontológico a pacientes portadores de deficiência. [mestrado]. São Paulo: FOUSP; 2002.
8. Mugayar LRF. Pacientes Portadores de Necessidades Especiais: Manual de Odontologia e Saúde Oral. $1^{\text {a }}$ ed. São Paulo: Pancast; 2000.

9. Botazzo C. Saúde Bucal e Cidadania.Transitando entre a teoria e a prática. 2003.

10. Minayo MCS. O desafio do conhecimento: Pesquisa Qualitativa em Saúde. $7^{a}$.ed. São Paulo: Hucitec; 2000.

11. Bardin L. Análise de Conteúdo. Lisboa: Edições 70; 1979.

12. Alves EGR, Ramos DLP.Profissionais de saúde: vivendo e convivendo com HIV/ AIDS. São Paulo: Santos; 2002.

13. Cardoso VES; Pernambuco RA, Olympio KPK, Bardal PAP, Silva PA. Programa odontológico de educação e prevenção em pacientes especiais: introdução dos educadores e auxiliares como higienistas. Odontologia e Sociedade 2002; 4(1): 49.

14. Jacob LS, Marchioni SAE, Galli VL. Algumas Contribuições da psicologia no atendimento odontológico ao paciente especial. In: Pacientes Portadores de Necessidades Especiais: Manual de Odontologia e Saúde Oral. $1^{\mathrm{a}}$ ed. São Paulo: Pancast; 2000.

15. Mello MASR. Autismo: Guia prático. $4^{\mathrm{a}}$ ed. São Paulo: AMA; Brasília: CORDE; 2004.

16. Seger L et al.. Psicologia \& Odontologia: uma abordagem integradora. $3 .^{\mathrm{a}}$ ed. São Paulo: Santos; 1998.

Recebido em 06 de novembro de 2009 Modificado em 19 de dezembro de 2009 Aceito em 23 de janeiro de 2010 